\title{
Adaptive Fuzzy Tracking Control for a Class of Uncertain Nonlinear Time-Delayed Systems with Saturation Constrains
}

\author{
Yu-Jun Zhang, ${ }^{1}$ Li-Bing Wu, ${ }^{2}$ Hong-Yang Zhao, ${ }^{3}$ Xiao-Dong Hu, ${ }^{3}$ \\ Wen-Yu Zhang, ${ }^{4}$ and Dong-Ying $\mathrm{Ju}^{3}$ \\ ${ }^{1}$ Software School, University of Science and Technology Liaoning, Anshan, Liaoning 114051, China \\ ${ }^{2}$ School of Science, University of Science and Technology Liaoning, Anshan, Liaoning 114051, China \\ ${ }^{3}$ School of Material and Metallurgy, University of Science and Technology Liaoning, Anshan, Liaoning 114051, China \\ ${ }^{4}$ College of International of Finance and Banking, University of Science and Technology Liaoning, Anshan, Liaoning 114051, China
}

Correspondence should be addressed to Dong-Ying Ju; dyju@sit.ac.jp

Received 28 September 2016; Revised 15 November 2016; Accepted 22 November 2016

Academic Editor: Xian Zhang

Copyright (c) 2016 Yu-Jun Zhang et al. This is an open access article distributed under the Creative Commons Attribution License, which permits unrestricted use, distribution, and reproduction in any medium, provided the original work is properly cited.

\begin{abstract}
In this paper, the problem of adaptive fuzzy tracking control is considered for a class of uncertain nonaffine nonlinear systems with external disturbances, multiple time delays, and nonsymmetric saturation constrains. First, the mean value theorem is employed to deal with the nonaffine term with input nonlinearity. Then, a new adaptive fuzzy tracking controller with parameter updating laws is designed by using fuzzy approximation technique. Moreover, it is shown that all the closed-loop signals are bounded and the tracking errors can asymptotically converge to zero via the Lyapunov stability analysis. Finally, the simulation example for van der Pol oscillator system is worked out to verify the effectiveness of the proposed adaptive fuzzy design approach.
\end{abstract}

\section{Introduction}

Over the past decades, the modeling and control design problem of nonlinear systems have attracted considerable attentions because of the extensively practical applications. Consequently, a large number of successful control schemes for uncertain nonlinear systems with dead-zone, time delays, and actuator failures have been developed in this area; see [1-13] and the references therein. More specifically, Tong and Li [1] studied the adaptive fuzzy tracking control problem for a class of nonlinear systems with dead-zone nonlinearities. In [9], by employing a nonlinear fault estimator, the output fault-tolerant tracking control was developed using the adaptive backstepping technique. Zhou et al. [13] considered the adaptive output tracking control problem for a class of nonlinear systems with stochastic disturbances and time delays. Recently, the authors in [14] proposed the adaptive tracking control approaches for a class of nonlinear timedelayed systems with dead-zone nonlinearities. The global stabilization problem for a class of nonlinear time-delayed systems [15] was considered by using multiswitching-based adaptive neural network control method. Besides, by combining fuzzy approximation and adaptive backstepping technique, a novel robust fault-tolerant control scheme [16] was developed for a class of non-lower-triangular nonlinear systems with actuator failures.

It should be pointed out that all the abovementioned control strategies are merely suitable for the considered nonlinear systems in affine form rather than nonaffine form. It is well known that the nonaffine nonlinear systems represent more general cases which can describe many practical processes. Li and Tong [17] proposed an adaptive fuzzy output control approach for a class of pure-feedback nonlinear systems with dead-zone constrains. In [18], an adaptive fuzzy asymptotic tracking controller was designed for a class of uncertain nonaffine nonlinear systems with dead-zone inputs. Meanwhile, the actuator saturation constrain control implies that the input signals are always bounded for most of practical systems. The saturation problem is very important for the actuator control design, and the performance and 
stability of the closed-loop systems will be severely effective if the input constrain in the design process is ignored. Thus, the adaptive control design for nonlinear systems with saturation constrains is a challenging topic. Wen et al. [19] studied the adaptive control problem for a class of uncertain nonlinear systems with saturation constrains. Based on the disturbance observer, direct adaptive NNs control strategies in [20] were developed for a class of uncertain nonaffine nonlinear systems with saturation inputs. Additionally, the adaptive fuzzy tracking control scheme for a class of nonaffine nonlinear systems with saturation constrains and stochastic disturbances in [21] was proposed.

Motivated by the above considerations, this paper studies the problem of adaptive fuzzy tracking control for a class of uncertain nonaffine nonlinear systems with input saturations. Compared with the existing results, the main contributions of this paper are as follows: (1) The approximation-based adaptive tracking control scheme is extended to nonaffine nonlinear systems with multiple time delays and saturation constrains. (2) Different from the design methods proposed in $[20,21]$, it is obtained that the tracking errors can asymptotically converge to zero rather than being uniformly ultimately bounded. (3) The mean value theorem is used to deal with the nonaffine term with input saturation, and thus the desired asymptotic tracking performance of the closedloop systems can be achieved by using Lyapunov stability analysis.

The rest of the paper is organized as follows. Section 2 gives the problem statement and preliminaries. A novel adaptive fuzzy asymptotic tracking controller is designed in Section 3. Simulation studies are then provided in Section 4 to verify the effectiveness of the proposed control method, and Section 5 draws the conclusions.

\section{Problem Statement and Preliminaries}

Consider the nonaffine nonlinear systems described as follows:

$$
\begin{gathered}
\dot{x}_{1}=x_{2} \\
\dot{x}_{2}=x_{3} \\
\vdots \\
\dot{x}_{n}=f(x, \text { sat }(u))+\sum_{i=1}^{r} \Delta E_{i}(x, t) x\left(t-\tau_{i}(t)\right)+d(t),
\end{gathered}
$$

where $x=\left[x_{1}, x_{2}, \ldots, x_{n}\right]^{T} \in \mathbb{R}^{n}$ is the system state, $u \in \mathbb{R}$ is the actual control input, and $d(t) \in \mathbb{R}$ is the unknown bounded external disturbance. The unknown nonlinear function $f(\cdot, \cdot): \mathbb{R}^{n} \times \mathbb{R} \rightarrow \mathbb{R}$ is sufficiently smooth. Without loss of generality, $\Delta E_{i}(\cdot), i=1,2, \ldots, r$, represent the system uncertainties and are assumed to be continuous with the approximate dimensions. $\tau_{i}(t)$ is the time-varying time delay satisfying $0 \leq \tau_{i}(t) \leq \tau_{i}^{*}, \dot{\tau}_{i}(t) \leq \bar{\tau}_{i}^{*}<1, i=1,2, \ldots, r$, $x(t)=\phi(t), t \in\left[0, \bar{\tau}^{*}\right]$, where $\phi(t)$ is the initial condition and $\bar{\tau}^{*}=\max _{1 \leq i \leq r}\left\{\tau_{i}^{*}\right\}$. In addition, sat $(u)$ denotes the input saturation, which is defined by

$$
\operatorname{sat}(u(t))= \begin{cases}u_{M}, & u(t) \geq u_{M}, \\ u(t), & u_{m} \leq u(t) \leq u_{M}, \\ u_{m}, & u(t) \leq u_{m},\end{cases}
$$

where $u_{M}$ and $u_{m}$ are known upper and lower bounds of the control output $u(t)$, respectively.

Moreover, as in [21], (2) can be written as

$$
\text { sat }(u(t))=S(u(t))+D(u(t)) \text {, }
$$

where the piecewise smooth function $S(u)$ is defined as

$$
\begin{aligned}
S(u) & = \begin{cases}u_{M} * \tanh \left(\frac{u}{u_{M}}\right), & u \geq 0, \\
u_{m} * \tanh \left(\frac{u}{u_{m}}\right), & u<0,\end{cases} \\
& = \begin{cases}u_{M} * \frac{e^{u / u_{M}}-e^{-u / u_{M}}}{e^{u / u_{M}}+e^{-u / u_{M}}}, & u \geq 0, \\
u_{m} * \frac{e^{u / u_{m}}-e^{-u / u_{m}}}{e^{u / u_{m}}+e^{-u / u_{m}}}, & u<0\end{cases}
\end{aligned}
$$

and $D(u)=\operatorname{sat}(u)-S(u)$. Furthermore, the bound of $D(u)$ can be obtained as

$$
\begin{aligned}
|D(u)| & \leq|\operatorname{sat}(u)-S(u)| \\
& \leq \max \left\{u_{M}(1-\tanh (1)), u_{m}(\tanh (1)-1)\right\} \\
& =\bar{D}
\end{aligned}
$$

with $\bar{D}$ being the upper bound between the continuous function $\operatorname{sat}(u)$ and the smooth function $S(u)$. Consequently, it follows from the mean value theorem that $S(u)=\left(\partial S\left(u_{\xi}\right) /\right.$ $\partial u) u+S(0)$ with $u_{\xi}=\lambda u$ and $0<\lambda<1$. Noting (3) and (4), we can obtain that

$$
\operatorname{sat}(u)=\frac{\partial S\left(u_{\xi}\right)}{\partial u} u+D(u)
$$

The control objective is to design an adaptive fuzzy asymptotic tracking controller $u(t)$ to ensure that all the closed-loop signals are bounded and the system states asymptotically stable in the presence of external disturbances, multiple time delays, and saturation constrains. Besides, in order to design adaptive state feedback controller, $x=\left[x_{1}, x_{2}\right.$, $\left.\ldots, x_{n}\right]^{T}$ is assumed to be measurable, and the state tracking error is defined as $e=x-x_{d}$ with the reference signal $x_{d}$ being $x_{d}=\left[y_{d}, \dot{y}_{d}, \ldots, y_{d}^{(n-1)}\right]^{T}$. Moreover, the following assumptions are given for system (1).

Assumption 1. There exist unknown positive constants $\bar{d}$ and $\bar{d}_{k}$ such that $|d(t)| \leq \bar{d}_{0}$ and $\left|y_{d}^{(k)}\right| \leq \bar{d}_{k}$ for $k=0,1,2, \ldots, n$, respectively. 
Assumption 2. The uncertain function $\Delta E_{i}(x, t)$ is assumed to satisfy $\left\|\Delta E_{i}(x, t)\right\| \leq \epsilon_{k}^{*}$ with $\epsilon_{i}^{*}, i=1,2, \ldots, r$, being unknown positive constants.

Assumption 3 (see $[18,20]$ ). For all $x \in \mathbb{R}^{n}$ and $u \in \mathbb{R}$ in system (5), there always exist positive constants $M_{1}$ and $M_{2}$ such that the following inequality holds:

$$
0<M_{1} \leq \frac{\partial f(x, u)}{\partial u} \leq M_{2}
$$

Assumption 4. Given the practical system described by (1) satisfying the input saturation (2), there exists feasible actual control input $u$, which can achieve the desired control objective.

Remark 5. Clearly, Assumption 1 is quite standard and means that the external disturbance, the reference output signal, and its time derivatives are bounded, respectively. It follows from Assumption 2 that the change rate of the input gain is bounded. Particularly, different from [20, 21], the tracking error of this paper can asymptotically converge to zero rather than to a desired compact set.

Similar to $[10,11,16-18]$, the following fuzzy approximation lemma is given by the following lemma.

Lemma 6. Let $F(X)$ be a continuous function that is defined on a compact set $\Omega_{X}$. For any given positive constant $\epsilon$, there always exists a fuzzy logic system $y(X)$ in the form of (7) such that

$$
\sup _{X \in \Omega_{X}}|F(X)-y(X)|=\left|F(X)-\theta^{T} \xi(X)\right|<\epsilon .
$$

Consequently, the optimal parameter vectors $\theta^{*}$ of fuzzy logic system (FLS) is defined as

$$
\theta^{*}=\arg \min _{\theta \in \Omega_{\theta}}\left[\sup _{X \in \Omega_{X}}\left|F(X)-\theta^{T} \xi(X)\right|\right],
$$

where $\Omega_{\theta}$ and $\Omega_{X}$ are compact regions for $\theta$ and $X$, respectively. In addition, the fuzzy approximation error $\delta^{*}(X)$ is defined as

$$
F(X)=\theta^{* T} \xi(X)+\delta^{*}(X), \quad \forall X \in \Omega_{X}, \theta^{*} \in \Omega_{\theta} .
$$

\section{Adaptive Fuzzy Tracking Controller Design}

In this section, the adaptive fuzzy asymptotic tracking control scheme will be developed for the nonlinear system (1) with external disturbance, multiple time delays, and saturation constrain. For this purpose, taking the time derivative of the tracking error $e=x-x_{d}$ with respect to $t$ yields

$$
\begin{aligned}
\dot{e}= & A e+B(f(x, \text { sat }(u)) \\
& \left.+\sum_{i=1}^{r} \Delta E_{i}(x, t) x\left(t-\tau_{i}(t)\right)+d(t)-x_{d}^{(n)}\right),
\end{aligned}
$$

where

$$
A=\left[\begin{array}{ccccc}
0 & 1 & 0 & \cdots & 0 \\
0 & 0 & 1 & \cdots & 0 \\
& \ddots & & \ddots & \\
0 & 0 & 0 & \cdots & 1 \\
0 & 0 & 0 & \cdots & 0
\end{array}\right],
$$$$
B=\left[\begin{array}{c}
0 \\
0 \\
\vdots \\
0 \\
1
\end{array}\right] .
$$

Then, from (12), $A+B K$ is a stable matrix by properly choosing a gain vector $K$. Moreover, for any given $Q=Q^{T}>0$, there exists $P=P^{T}>0$ such that the Lyapunov equation $(A+$ $B K)^{T} P+P(A+B K)+(r / \eta) I=-Q$ holds, where $\eta$ is a positive design parameter.

For the nonaffine term $f(x, \operatorname{sat}(u))$, using the mean value theorem again gives $f(x, \operatorname{sat}(u))=f(x, 0)+(\partial f(x$, $\left.\left.u_{\zeta}\right) / \partial u\right) \operatorname{sat}(u)$ with $u_{\zeta}=\mu u$ and $0<\mu<1$. From (6), it is easy to see that

$$
\begin{aligned}
f(x, \text { sat }(u))= & f(x, 0) \\
& +\frac{\partial f\left(x, u_{\zeta}\right)}{\partial u}\left(\frac{\partial S\left(u_{\xi}\right)}{\partial u} u+D(u)\right) \\
= & \tau_{s} u+\Psi(x, u),
\end{aligned}
$$

where $\Psi(x, u)=\left(\left(\partial f\left(x, u_{\zeta}\right) / \partial u\right)\left(\partial S\left(u_{\xi}\right) / \partial u\right)-\tau_{s}\right) u+f(x, 0)+$ $D(u)$ with $\tau_{s}$ being a positive design parameter. In addition, by using the expression in (10), (13) can be expressed as

$$
\begin{aligned}
f(x, \text { sat }(u))=\tau_{s} u+\theta^{* T} \xi(x, e)+ & \delta^{*}(x, e), \\
& \forall(x, e) \in \Omega \subset \mathbb{R}^{2 n},
\end{aligned}
$$

where the approximation error $\delta^{*}(x, e)$ satisfies $\left|\delta^{*}(x, e)\right| \leq$ $\delta^{*}$ with $\delta^{*}$ being any small positive constant, and $\Omega$ is an appropriate compact set. Without loss of generality, based on Assumptions 1 and 2, we introduce the notions $K_{1}^{*}=$ $\sup _{t \geq 0}\left(\left|x_{d}^{(n)}(t)\right|+|d(t)|+\sum_{i=1}^{k}\left\|x_{d}\left(t-\tau_{i}(t)\right)\right\|+\delta^{*}\right)$ and $K_{2}^{*}=$ $\sum_{i=1}^{k}\left(\epsilon_{i}^{* 2} /\left(1-\bar{\tau}_{i}^{*}\right)\right)$, where $K_{1}^{*}$ and $K_{2}^{*}$ are unknown constants. Therefore, the adaptive fuzzy controller is designed as

$$
\begin{aligned}
u= & \tau_{s}^{-1}\left(K e-\widehat{\theta}^{T} \xi(x, e)-\frac{\widehat{K}_{1}^{2} B^{T} P e}{\left|e^{T} P B\right| \widehat{K}_{1}+\sigma(t)}\right. \\
& \left.-\frac{1}{2} \eta B^{T} P e \widehat{K}_{2}\right)
\end{aligned}
$$


with the corresponding adaptive control laws

$$
\begin{gathered}
\dot{\hat{\theta}}=-\Gamma \sigma \widehat{\theta}-\Gamma e^{T} P B \xi(x, e), \\
\dot{\widehat{K}}_{1}=-\gamma_{1} \sigma \widehat{K}_{1}+2 \gamma_{1}\left\|B^{T} P e\right\|, \\
\dot{\widehat{K}}_{2}=-\gamma_{2} \sigma \widehat{K}_{2}+\gamma_{2} \eta\left\|B^{T} P e\right\|^{2},
\end{gathered}
$$

where $\widehat{K}_{i}$ and $\widehat{\theta}$ are the estimates of $K_{i}^{*}$ and $\theta^{*}$, respectively; $\Gamma=\Gamma^{T}>0$ and $\gamma_{i}$ are positive design parameters. Besides, the continuous function $\sigma(t)$ is subject to $\sigma(t)>0$ and $\int_{0}^{t} \sigma(\tau) d \tau \leq \bar{\sigma}<\infty, \forall t \geq 0$, with any constant $\bar{\sigma}>0, i=1,2$.

Remark 7. The adaptive fuzzy controller (15) mainly consists of four terms. Concretely, $\tau_{s}^{-1}$ is the positive design parameter of the adaptive control gain, and the first term $K x$ of the right hand side plays a key role for stabilizing system. The second term is used to decouple the nonaffine term with saturation nonlinearity. The third term and the fourth term with adaptation laws (16) are used to deal with the effects of multiple time delays and external disturbance, respectively.

Now, the stability of the resulting closed-loop system is given in the following theorem.

Theorem 8. Consider the uncertain nonaffine nonlinear system (1) satisfying Assumptions 1-4. With the application of adaptive fuzzy controller (15) and parameter updated laws (16), the tracking error of the closed-loop system can asymptotically converge to zero; that is, $\lim _{t \rightarrow \infty} e(t)=0$ for any $(x, e) \in \Omega$, which is a proper compact set.

Proof. For the closed-loop error system (11), choose a Lyapunov function candidate as follows:

$$
\begin{aligned}
V\left(e, \widetilde{\theta}, \widetilde{K}_{1}, \widetilde{K}_{2}\right)= & e^{T} P e+\frac{1}{2} \sum_{i=1}^{2} \gamma_{i}^{-1} \widetilde{K}_{i}^{2}+\frac{1}{2} \widetilde{\theta}^{T} \Gamma^{-1} \widetilde{\theta} \\
& +\eta^{-1} \sum_{i=1}^{r} \int_{t-\tau_{i}(t)}^{t} e^{T}(s) e(s) d s,
\end{aligned}
$$

where $\widetilde{\theta}=\widehat{\theta}-\theta^{*}$ and $\widetilde{K}_{i}=\widehat{K}_{i}-K_{i}^{*}, i=1,2$, are the parameter estimation errors. Then, taking the time derivative of $V$ with respect to $t$ yields

$$
\begin{aligned}
\dot{V} & =e^{T}\left(P A+A^{T} P\right) e+\sum_{i=1}^{2} \gamma_{i}^{-1} \widetilde{K}_{i} \dot{\widetilde{K}}_{i}+\widetilde{\theta}^{T} \Gamma^{-1} \dot{\tilde{\theta}} \\
& +2 e^{T} P B(f(x, \text { sat }(u)) \\
& \left.+\sum_{i=1}^{r} \Delta E_{i}(x, t) x\left(t-\tau_{i}(t)\right)+d(t)-x_{d}^{(n)}\right) \\
& +\eta^{-1} \sum_{i=1}^{r}\left(\|e(t)\|^{2}-\left(1-\dot{\tau}_{i}(t)\right)\left\|e\left(t-\tau_{i}(t)\right)\right\|^{2}\right) .
\end{aligned}
$$

By invoking (14), we obtain that

$$
\begin{aligned}
\dot{V} & =e^{T}\left(P A+A^{T} P\right) e \\
& +2 e^{T} P B \sum_{i=1}^{r} \Delta E_{i}(x, t) x\left(t-\tau_{i}(t)\right)+2 e^{T} P B\left(\tau_{s} u\right. \\
& \left.+\theta^{* T} \xi(x, e)+\delta^{*}(x, e)+d(t)-x_{d}^{(n)}\right) \\
& +\eta^{-1} \sum_{i=1}^{r}\left(\|e(t)\|^{2}-\left(1-\dot{\tau}_{i}(t)\right)\left\|e\left(t-\tau_{i}(t)\right)\right\|^{2}\right) \\
& +\sum_{i=1}^{2} \gamma_{i}^{-1} \widetilde{K}_{i} \dot{\widetilde{K}}_{i}+\widetilde{\theta}^{T} \Gamma^{-1} \dot{\tilde{\theta}} \leq e^{T}\left(P A+A^{T} P\right) e \\
& +2 \tau_{s} e^{T} P B u+2 e^{T} P B \theta^{* T} \xi(x, e)+2\left|e^{T} P B\right| \\
& \cdot \mid \delta^{*}(x, e)+d(t)-x_{d}^{(n)} \\
& +\sum_{i=1}^{r} \Delta E_{i}(x, t) x_{d}\left(t-\tau_{i}(t)\right) \mid+\frac{r}{\eta}\|e(t)\|^{2} \\
& -\sum_{i=1}^{r}\left(1-\bar{\tau}_{i}^{*}\right)\left\|e\left(t-\tau_{i}(t)\right)\right\|^{2}+2\left|e^{T} P B\right| \\
& +\sum_{i=1}^{r} \epsilon_{i}^{*}\left\|e\left(t-\tau_{i}(t)\right)\right\|+\sum_{i=1}^{2} \gamma_{i}^{-1} \widetilde{K}_{i} \dot{\widetilde{K}}_{i}+\widetilde{\theta}^{T} \Gamma^{-1} \dot{\tilde{\theta}} .
\end{aligned}
$$

Using triangle inequality and according to the definitions of $K_{i}^{*}, i=1,2$,

$$
\begin{aligned}
\dot{V} \leq & e^{T}\left(P A+A^{T} P\right) e+2 \tau_{s} e^{T} P B u \\
& +2 e^{T} P B \theta^{* T} \xi(x, e)+2\left|e^{T} P B\right| K_{1}^{*}+\frac{r}{\eta}\|e(t)\|^{2} \\
& +\eta\left|e^{T} P B\right| K_{2}^{*}+\sum_{i=1}^{2} \gamma_{i}^{-1} \widetilde{K}_{i} \dot{\widetilde{K}}_{i}+\widetilde{\theta}^{T} \Gamma^{-1} \dot{\tilde{\theta}} .
\end{aligned}
$$

Then, from the adaptive controller (15) and the parameter updated laws (16), we can obtain that

$$
\dot{V} \leq-e^{T} Q e+\frac{2\left|e^{T} P B\right| \widehat{K}_{1} \sigma}{\left|e^{T} P B\right| \widehat{K}_{1}+\sigma}-\sigma \widetilde{\theta}^{T} \widehat{\theta}-\sigma \sum_{i=1}^{2} \widetilde{K}_{i} \widehat{K}_{i} .
$$

Using the inequality $0 \leq x /(x+y)<1, \forall x \geq 0, y>0$, and $a^{T} b \leq\|a\|\|b\| \forall a, b \in \mathbb{R}^{n}$, (21) becomes

$$
\begin{aligned}
\dot{V} & \leq-e^{T} Q e+\sigma\left(\frac{\theta^{* 2}}{4}+\frac{K_{1}^{* 2}}{4}+\frac{K_{2}^{* 2}}{4}+2\right) \\
& =-e^{T} Q e+\sigma \pi,
\end{aligned}
$$

where $\pi=\theta^{* 2} / 4+K_{1}^{* 2} / 4+K_{2}^{* 2} / 4+2$.

Integrating (22) from 0 to $t$ yields

$$
V(t)+\int_{0}^{t} e^{T}(\tau) Q e(\tau) d \tau \leq\left. V\right|_{t=0}+\pi \bar{\sigma} .
$$


Thus, it further implies that $\int_{0}^{t} e^{T}(\tau) e(\tau) d \tau \leq$ (1/ $\left.\lambda_{\text {min }}(Q)\right)\left(\left.V\right|_{t=0}+\pi \bar{\sigma}\right), \forall t>0$, where $\lambda_{\text {min }}(\cdot)$ denotes the minimum eigenvalue of a matrix, that is, $e \in L_{2}$. According to Barbalat's lemma [22], it can be concluded that $\lim _{t \rightarrow \infty} e(t)=$ 0 . The proof is completed.

Remark 9. It should be pointed out that the control methods proposed in $[20,21]$ can guarantee that the tracking errors converge to the desired compact sets. The tracking error of the closed-loop system can asymptotically converge to zero by employing the adaptive control scheme in [18]; however, this control scheme cannot deal with nonaffine nonlinear systems with multiple time delays and saturation constrains. In this paper, based on fuzzy approximation technique and the mean value theorem, the proper nonlinear functions, it is proved that the desired asymptotic tracking performance of the closed-loop systems can be achieved via Lyapunov stability analysis.

\section{Simulation Studies}

In this section, a third-order van der Pol oscillator system from [23] is used for simulation study of this paper. Besides, by adding the external disturbances $d(t)$ and the multiple time-delayed perturbation $\sum_{i=1}^{r} \Delta E_{i}(x, t) x\left(t-\tau_{i}(t)\right)$, the corresponding nonaffine nonlinear system model is described as follows:

$$
\begin{aligned}
\dot{x}_{1}= & x_{2} \\
\dot{x}_{2}= & x_{3} \\
\dot{x}_{3}= & -x_{1}-0.5 x_{2}+\beta\left(1-\left(x_{1}+0.5 x_{2}\right)^{2}\right) x_{3} \\
& +\left(2+\sin \left(x_{1} x_{2} x_{3}\right)\right)\left(u+\frac{1}{3} u^{3}+\sin (u)\right) \\
& +\sum_{i=1}^{r} \Delta E_{i}(x, t) x\left(t-\tau_{i}(t)\right)+d(t) \\
y= & x_{1},
\end{aligned}
$$

where $\beta=0.7$, the time delays and parameter uncertain functions are $\tau_{1}(t)=0.8 \cos (t), \tau_{2}(t)=0.5 \sin (t), \tau_{3}(t)=$ $0.5 \sin (2 t)$ and $E_{1}(x, t)=[1,0,0], E_{2}(x, t)=[0,2,0]$, $E_{3}(x, t)=[0,0,3]$, and the external disturbance $d(t)$ is chosen as $d(t)=0.5 \sin (t)$, respectively, and the reference signal is given as $y_{d}=0.1+\sin (0.5 t)$.

Take the membership functions of fuzzy logic system as follows:

$$
\begin{aligned}
& \mu_{F_{j}^{1}}=\exp \left[-\frac{\left(x_{j}+1.5\right)^{2}}{2}\right], \\
& \mu_{F_{j}^{2}}=\exp \left[-\frac{\left(x_{j}+1.25\right)^{2}}{2}\right], \\
& \mu_{F_{j}^{3}}=\exp \left[-\frac{\left(x_{j}+1\right)^{2}}{2}\right],
\end{aligned}
$$

$$
\begin{aligned}
& \mu_{F_{j}^{4}}=\exp \left[-\frac{\left(x_{j}+0.75\right)^{2}}{2}\right], \\
& \mu_{F_{j}^{5}}=\exp \left[-\frac{\left(x_{j}+0.5\right)^{2}}{2}\right], \\
& \mu_{F_{j}^{6}}=\exp \left[-\frac{\left(x_{j}+0.25\right)^{2}}{2}\right], \\
& \mu_{F_{j}^{7}}=\exp \left(-\frac{x_{j}^{2}}{2}\right), \\
& \mu_{F_{j}^{8}}=\exp \left[-\frac{\left(x_{j}-0.25\right)^{2}}{2}\right], \\
& \mu_{F_{j}^{9}}=\exp \left[-\frac{\left(x_{j}-0.5\right)^{2}}{2}\right], \\
& \mu_{F_{j}^{14}}=\exp \left[-\frac{\left(x_{j}-1.75\right)^{2}}{2}\right], \\
& \mu_{F_{j}^{10}}=\exp \left[-\frac{\left(x_{j}-0.75\right)^{2}}{2}\right], \\
& \mu_{F_{j}^{12}}=\exp \left[-\frac{\left(x_{j}-1.25\right)^{2}}{2}\right], \\
& =\exp \left[-\frac{\left(x_{j}-1\right)^{2}}{2}\right],
\end{aligned}
$$

Define fuzzy basis functions as

$$
\bar{\xi}_{i}(x)=\frac{\prod_{j=1}^{3} \mu_{F_{j}^{i}}\left(x_{j}\right)}{\sum_{i=1}^{14}\left[\prod_{j=1}^{3} \mu_{F_{j}^{i}}\left(x_{j}\right)\right]}, \quad i=1,2, \ldots, 14,
$$

where $x=\left[x_{1}, x_{2}, x_{3}\right]^{T}$.

By choosing gain $K=\left[\begin{array}{lll}-6 & -11 & -6\end{array}\right]$ and $Q=I>0$, it is easy to obtain that $P=\left[\begin{array}{lll}2.3617 & 1.4950 & 0.1083 \\ 1.4950 & 2.6108 \\ 0.1083 & 0.1950 & 0.1950 \\ 0.1408\end{array}\right]$. Based on Theorem 8 , the adaptive controller and the parameter updated laws are designed as (15) and (16), respectively. The corresponding simulation parameters are selected as $r=3, \eta=10, \epsilon=0.2$, $\mu_{i}=1, \Gamma_{0}=5 I, \sigma_{0}(t)=0.5 e^{-0.1 t}, \gamma_{1}=\gamma_{2}=1$, and the initial 


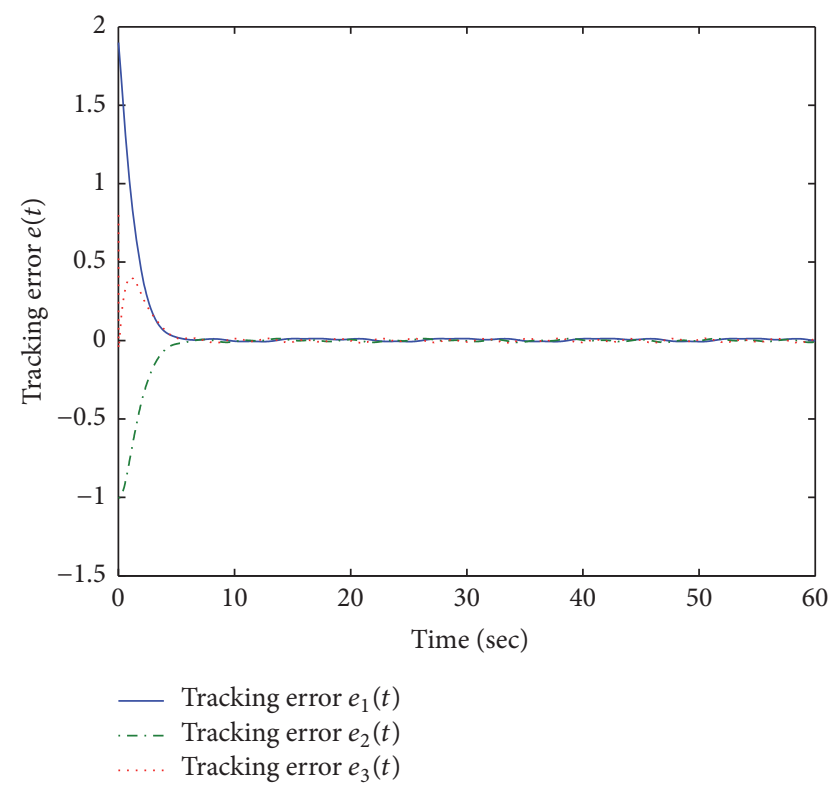

FIGURE 1: Trajectory of the tracking errors $e_{1}=y-x_{d}$ and $e_{2}=\dot{y}-\dot{x}_{d}$.

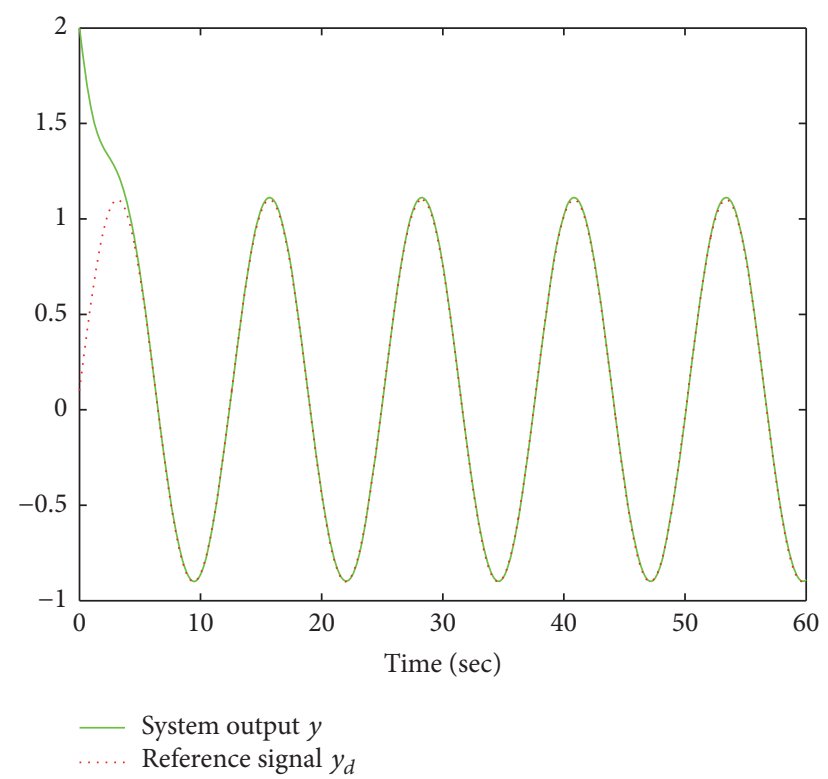

FIGURE 2: System output $y$ and desired output $x_{d}$.

values are chosen as $x(0)=[2,-0.5,0.8]^{T}, \widehat{\theta}(0)=[0,1,0,1$, $0,1,0,1,0,1,0,1,0,1]^{T}, \widehat{K}_{1}(0)=2, \widehat{K}_{2}(0)=6$. The simulation results are shown in Figures $1-7$. From Figures 1 and 2 , it can be seen that the state tracking errors converge to zero for the uncertain nonaffine nonlinear system (1) with multiple time delays, saturation constrain, and external disturbance simultaneously. Moreover, the boundedness of parameter estimations $\widehat{\theta}$ and $\widehat{K}_{j}, j=1,2$, is shown in Figures $3-5$. In Figures 6 and 7 , we can see that the actual control input and the saturation output signal are also bounded, respectively.

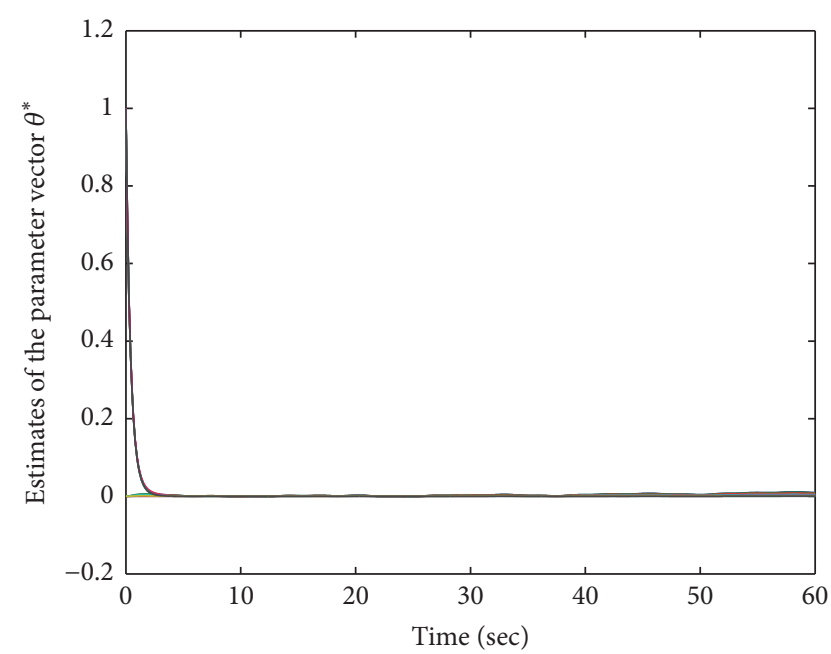

FIgURE 3: Response curves of $\widehat{\theta}$.

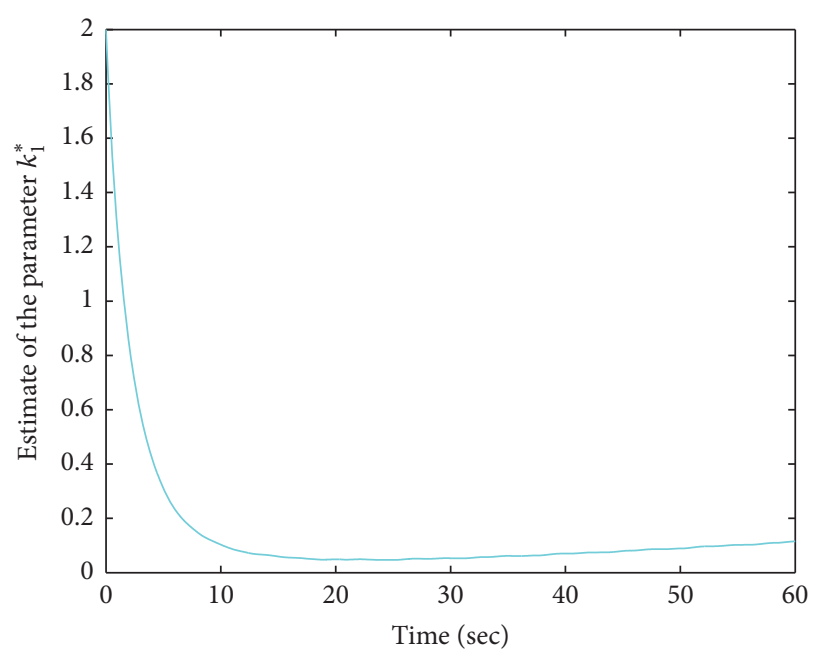

FIgURE 4: Response curve of $\widehat{K}_{1}$.

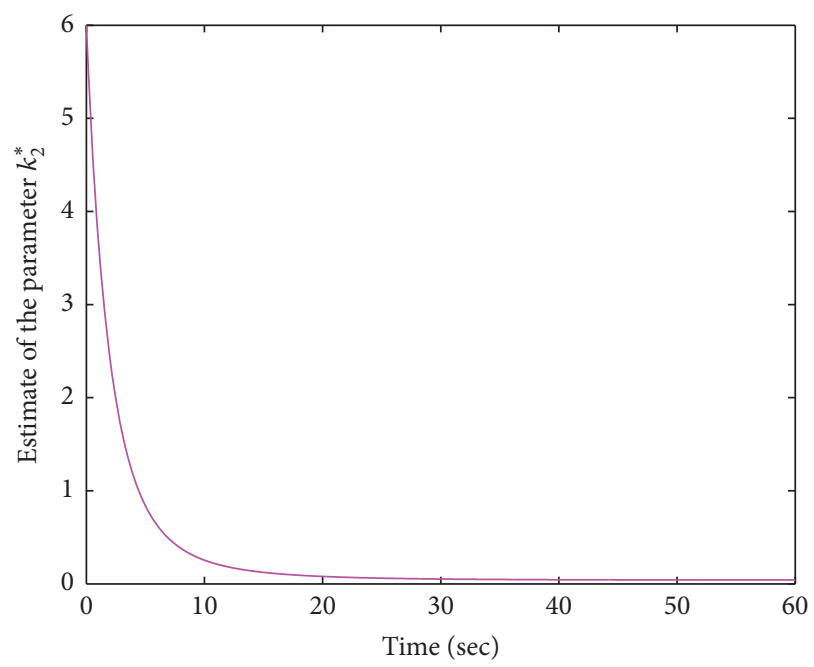

FIgURE 5: Response curve of $\widehat{K}_{2}$. 


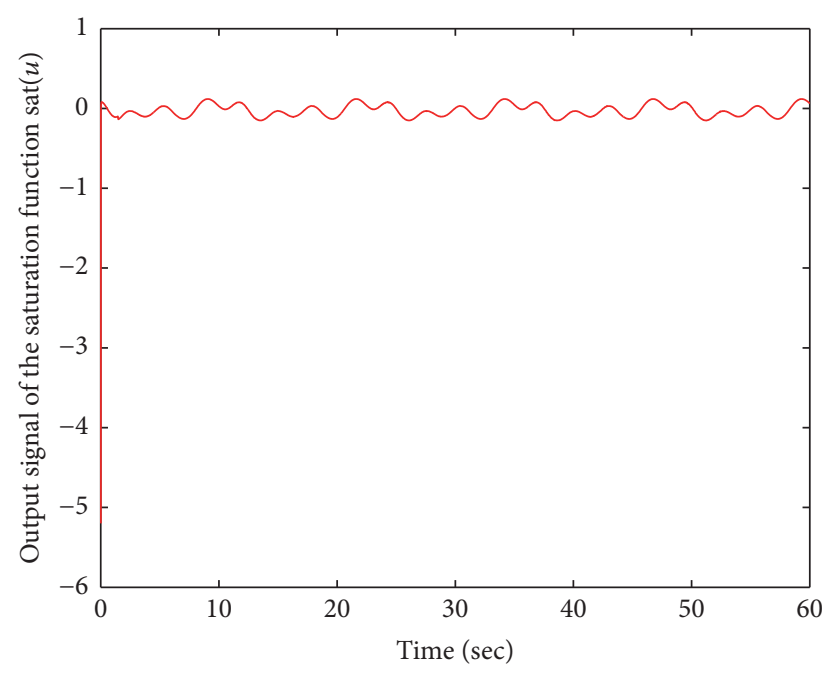

FIGURE 6: Output respond curve of the saturation function sat $(u)$.

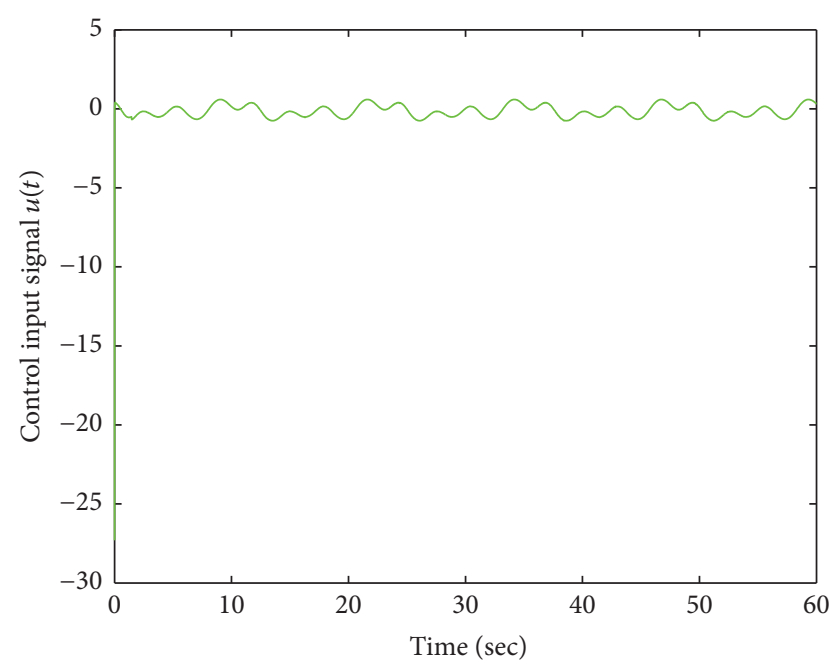

Figure 7: Respond curve of the control input $u(t)$.

\section{Conclusion}

This paper studies a novel adaptive fuzzy asymptotic tracking control scheme for a class of uncertain nonaffine nonlinear systems with multiple time delays, saturation constrains, and external disturbances. By using the mean value theorem and fuzzy logic system (FLS), the parameter updated laws are constructed to estimate the unknown adaptive controller parameters online. It is also shown that the proposed control method guarantees all the closed-loop system signals to be uniformly bounded and the tracking error can asymptotically converge to zero based on Lyapunov-based analysis. Numerical simulation results are provided to show the effectiveness of the proposed adaptive fuzzy tracking control design approach.

\section{Competing Interests}

The authors declare that they have no competing interests.

\section{Acknowledgments}

This work was supported in part by the Fundamental Research Funds of Anshan Municipal Government.

\section{References}

[1] S. Tong and Y. Li, "Adaptive fuzzy output feedback tracking backstepping control of strict-feedback nonlinear systems with unknown dead zones," IEEE Transactions on Fuzzy Systems, vol. 20, no. 1, pp. 168-180, 2012.

[2] X. Zhang, L. G. Wu, and S. C. Cui, "An improved integral inequality to stability analysis of genetic regulatory networks with interval time-varying delays," IEEE/ACM Transactions on Computational Biology and Bioinformatics, vol. 12, no. 2, pp. 398-409, 2015.

[3] X. Lin, X. Zhang, and Y. Wang, "Robust passive filtering for neutral-type neural networks with time-varying discrete and unbounded distributed delays," Journal of the Franklin Institute. Engineering and Applied Mathematics, vol. 350, no. 5, pp. 966989, 2013.

[4] X. Wang and G.-H. Yang, "Distributed fault-tolerant control for a class of cooperative uncertain systems with actuator failures and switching topologies," Information Sciences, vol. 370-371, pp. 650-666, 2016.

[5] X. Wang and G.-H. Yang, "Cooperative adaptive fault-tolerant tracking control for a class of multi-agent systems with actuator failures and mismatched parameter uncertainties," IET Control Theory \& Applications, vol. 9, no. 8, pp. 1274-1284, 2015.

[6] J.-W. Zhu and G.-H. Yang, "Fault accommodation for linear systems with time-varying delay," International Journal of Systems Science, vol. 48, no. 2, pp. 316-323, 2017.

[7] S. Tong and Y. Li, "Adaptive fuzzy output feedback control of MIMO nonlinear systems with unknown dead-zone inputs," IEEE Transactions on Fuzzy Systems, vol. 21, no. 1, pp. 134-146, 2013.

[8] Z. Zhang, S. Xu, and B. Zhang, "Asymptotic tracking control of uncertain nonlinear systems with unknown actuator nonlinearity," IEEE Transactions on Automatic Control, vol. 59, no. 5, pp. 1336-1341, 2014.

[9] M. Chen, B. Jiang, and W. W. Guo, "Fault-tolerant control for a class of non-linear systems with dead-zone," International Journal of Systems Science, vol. 47, no. 7, pp. 1689-1699, 2016.

[10] S. Tong, B. Huo, and Y. Li, “Observer-based adaptive decentralized fuzzy fault-tolerant control of nonlinear large-scale systems with actuator failures," IEEE Transactions on Fuzzy Systems, vol. 22, no. 1, pp. 1-15, 2014.

[11] S. C. Tong, T. Wang, and Y. M. Li, "Fuzzy adaptive actuator failure compensation control of uncertain stochastic nonlinear systems with unmodeled dynamics," IEEE Transactions on Fuzzy Systems, vol. 22, no. 3, pp. 563-574, 2014.

[12] Z. Zhang, S. Xu, and B. Zhang, "Asymptotic tracking control of uncertain nonlinear systems with unknown actuator nonlinearity," Institute of Electrical and Electronics Engineers. Transactions on Automatic Control, vol. 59, no. 5, pp. 1336-1341, 2014.

[13] Q. Zhou, P. Shi, S. Xu, and H. Li, "Observer-based adaptive neural network control for nonlinear stochastic systems with time delay," IEEE Transactions on Neural Networks and Learning Systems, vol. 24, no. 1, pp. 71-80, 2013.

[14] Z. Q. Zhang, S. Y. Xu, and B. Y. Zhang, "Exact tracking control of nonlinear systems with time delays and dead-zone input," Automatica, vol. 52, pp. 272-276, 2015. 
[15] J. Wu, W. Chen, F. Yang, J. Li, and Q. Zhu, "Global adaptive neural control for strict-feedback time-delay systems with predefined output accuracy," Information Sciences, vol. 301, pp. 2743, 2015.

[16] H. Wang, X. Liu, P. X. Liu, and S. Li, "Robust adaptive fuzzy fault-tolerant control for a class of non-lower-triangular nonlinear systems with actuator failures," Information Sciences, vol. 336, pp. 60-74, 2016.

[17] Y. Li and S. Tong, "Adaptive fuzzy output-feedback control of pure-feedback uncertain nonlinear systems with unknown dead zone," IEEE Transactions on Fuzzy Systems, vol. 22, no. 5, pp. 1341-1347, 2014.

[18] L.-B. Wu, G.-H. Yang, H. Wang, and F. Wang, "Adaptive fuzzy asymptotic tracking control of uncertain nonaffine nonlinear systems with non-symmetric dead-zone nonlinearities," Information Sciences, vol. 348, pp. 1-14, 2016.

[19] C. Wen, J. Zhou, Z. Liu, and H. Su, "Robust adaptive control of uncertain nonlinear systems in the presence of input saturation and external disturbance," IEEE Transactions on Automatic Control, vol. 56, no. 7, pp. 1672-1678, 2011.

[20] M. Chen and S. S. Ge, "Direct adaptive neural control for a class of uncertain nonaffine nonlinear systems based on disturbance observer," IEEE Transactions on Cybernetics, vol. 43, no. 4, pp. 1213-1225, 2013.

[21] H. Wang, B. Chen, X. Liu, K. Liu, and C. Lin, "Robust adaptive fuzzy tracking control for pure-feedback stochastic nonlinear systems with input constraints," IEEE Transactions on Cybernetics, vol. 43, no. 6, pp. 2093-2104, 2013.

[22] M. Krstic, I. Kanellakopoulos, and P. V. Kokotovic, Nonlinear and Adaptive Control Design, Wiley, New York, NY, USA, 1995.

[23] S.-L. Dai, C. Wang, and M. Wang, "Dynamic learning from adaptive neural network control of a class of nonaffine nonlinear systems," IEEE Transactions on Neural Networks and Learning Systems, vol. 25, no. 1, pp. 111-123, 2014. 


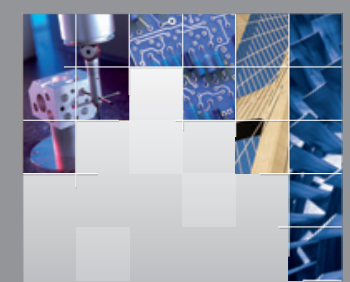

\section{Enfincering}
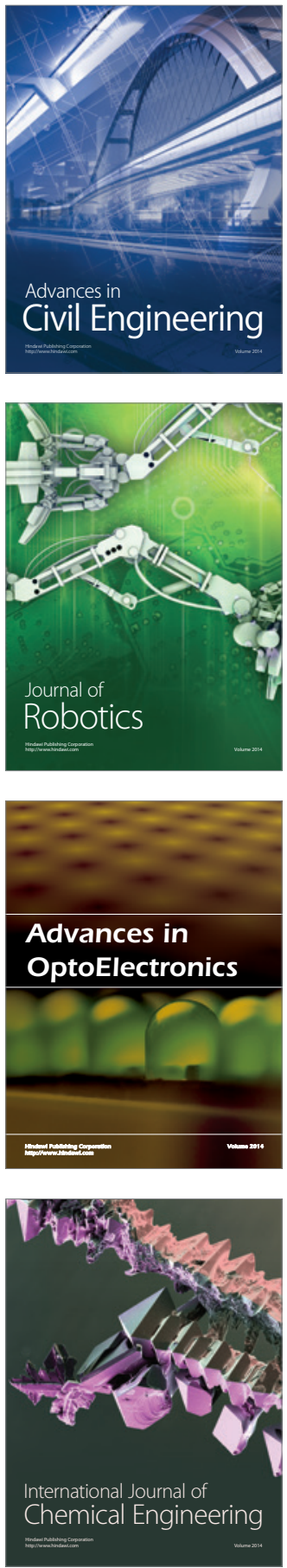

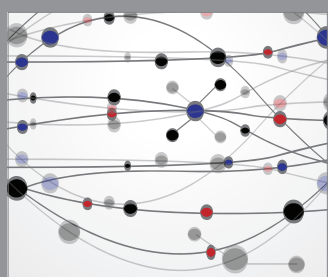

The Scientific World Journal

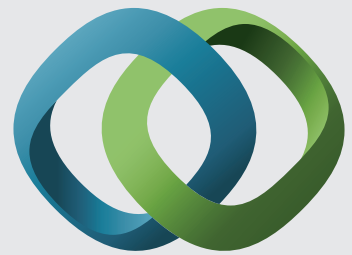

\section{Hindawi}

Submit your manuscripts at

http://www.hindawi.com
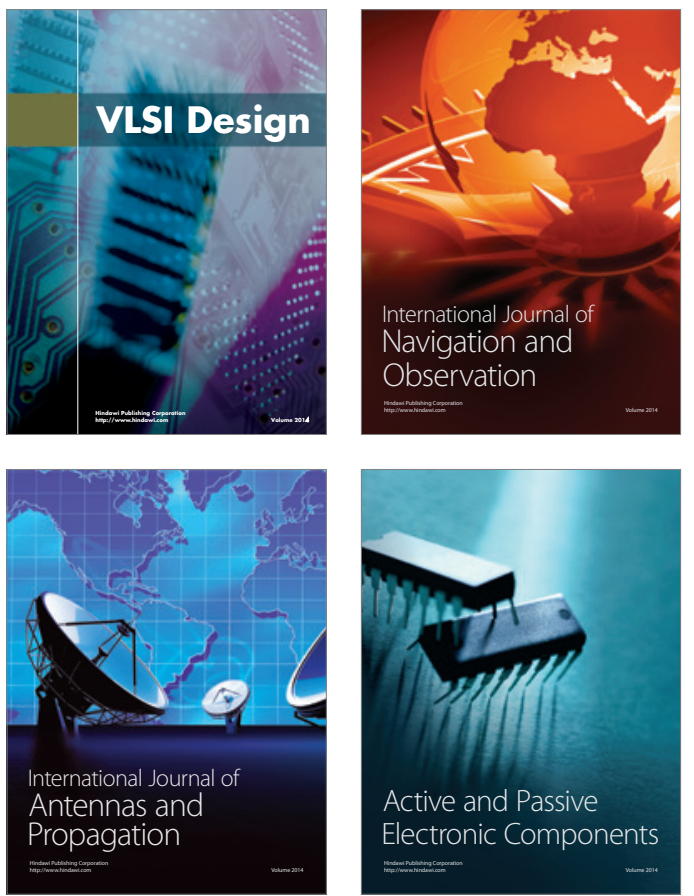
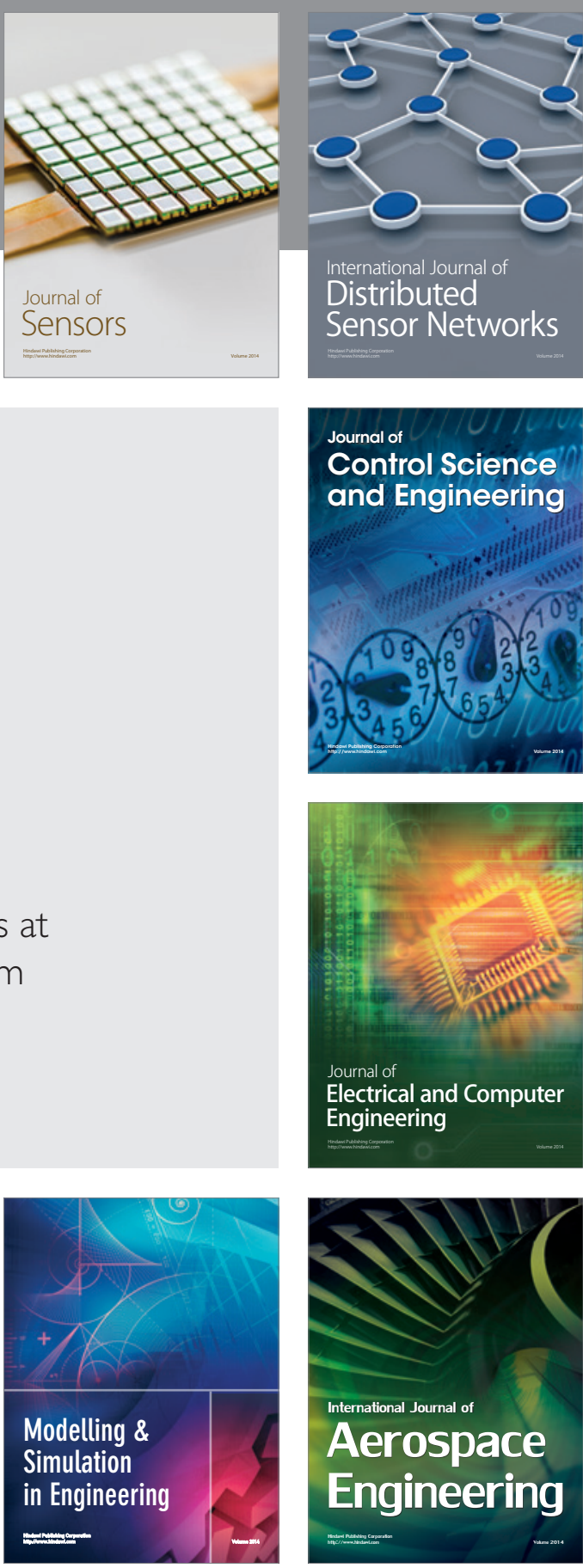

International Journal of

Distributed

Sensor Networks

Journal of

Control Science

and Engineering
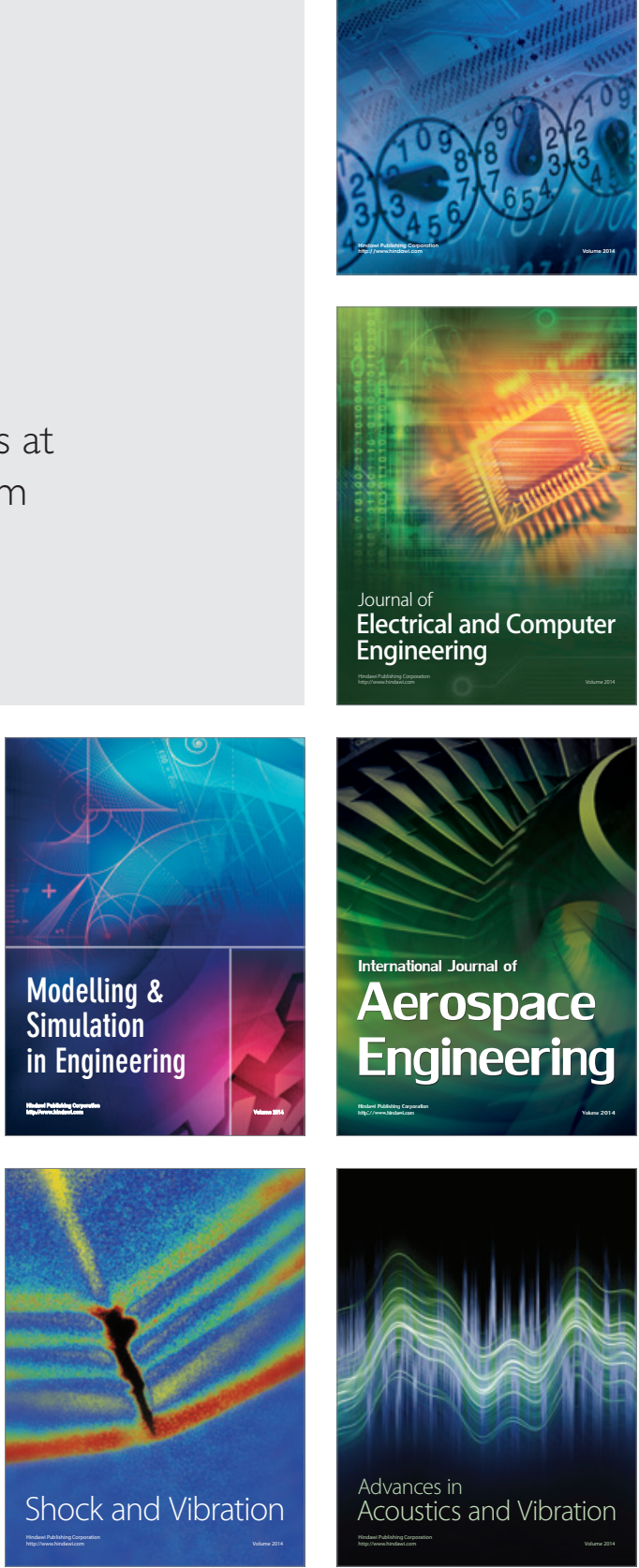